\title{
Generation of intense attosecond x-ray pulses using ultraviolet laser induced microbunching in electron beams
}

\author{
D. Xiang, Z. Huang, and G. Stupakov \\ SLAC National Accelerator Laboratory, Menlo Park, California 94025, USA
}

(Received 23 January 2009; published 2 June 2009)

\begin{abstract}
We propose a scheme that combines the echo-enabled harmonic generation technique with the bunch compression and allows one to generate harmonic numbers of a few hundred in a microbunched beam through up-conversion of the frequency of an ultraviolet seed laser. A few-cycle intense laser is used to generate the required energy chirp in the beam for bunch compression and for selection of an attosecond $\mathrm{x}$-ray pulse. Sending this beam through a short undulator results in an intense isolated attosecond x-ray pulse. Using a representative realistic set of parameters, we show that $1 \mathrm{~nm}$ x-ray pulse with peak power of a few hundred MW and duration as short as 20 attoseconds (FWHM) can be generated from a $200 \mathrm{~nm}$ ultraviolet seed laser. The proposed scheme may enable the study of electronic dynamics with a resolution beyond the atomic unit of time ( $\sim 24$ attoseconds) and may open a new regime of ultrafast sciences.
\end{abstract}

DOI: 10.1103/PhysRevSTAB.12.060701

PACS numbers: $41.60 . \mathrm{Cr}, 42.55 . \mathrm{Vc}$

\section{INTRODUCTION}

Fast time-dependent phenomena are typically studied with a pump-probe technique in which the dynamics are initiated by a pump laser and then probed by a delayed pulse. The temporal resolution depends on the duration of the pump and probe beams. The advent of attosecond (as) pulses made possible the time-resolved study of the electronic dynamics which opened up many new ultrafast sciences [1-5]. Using the $\sim 100$ as soft $\mathrm{x}$-ray pulse in the wavelength $\sim 10 \mathrm{~nm}$, the light wave was measured in [2] and the electron tunneling in atoms was observed in [3]. Most recently, the pulse duration has been pushed to about 80 as in the extreme ultraviolet wavelength [5]. These studies all relied on the technique of high harmonic generation in gas where an intense laser pulse was focused on an atomic gas jet and the high harmonic of the laser was generated and further used as the probe. However, it appears difficult to generate intense harmonic radiation with wavelength down to $1 \mathrm{~nm}$ or shorter with this technique.

To achieve atomic spatial resolution, the radiation wavelength needs to be pushed to $\sim 1 \mathrm{~nm}$ or shorter, and the isolated attosecond pulse is also highly desirable. There is a growing trend to provide such attosecond $\mathrm{x}$-ray pulses using free-electron lasers (FEL) [6-15]. Most of the proposed schemes utilized intense lasers to manipulate the electron beam energy and then selected a small part of the electrons to lase [8-13]. The schemes [7,9-14] that use self-amplified spontaneous emission (SASE) to generate attosecond x-ray pulse may suffer from statistic fluctuations because SASE originates from electron beam shot noise. An alternative scheme [8] which overcomes this problem is to use the high gain harmonic generation (HGHG) configuration $[16,17]$. But due to the relatively low up-frequency conversion efficiency, the HGHG scheme requires an intense seed signal in the wavelength of a few nm which is in principle obtainable but does not exist today.

In this paper, we propose a novel scheme which allows generation of intense isolated attosecond $\mathrm{x}$-ray in the wavelength $\sim 1 \mathrm{~nm}$ or shorter based on existed technologies. The scheme combines the echo-enabled harmonic generation (EEHG) technique $[18,19]$ with the bunch compression technique and allows harmonic numbers of a few hundred to be accessible that eventually enables the generation of $x$-ray radiation from an ultraviolet (UV) seed laser. The required energy chirp in the bunch compression is provided by a few-cycle intense infrared (IR) laser which finally assists in generation of an isolated attosecond $\mathrm{x}$-ray pulse. Using a representative realistic set of parameters, we show that $1 \mathrm{~nm}$ isolated $\mathrm{x}$-ray pulse with duration of about 20 as (FWHM) and peak power of about 180 MW can be generated in a short radiator with only 12 undulator periods using the proposed scheme. Since the generated attosecond $\mathrm{x}$-ray pulse is in tight synchronization with the few-cycle laser, it is straightforward to use them for ultrafast pumpprobe experiments.

\section{METHODS}

Our scheme requires an ultrarelativistic electron beam, a UV seed laser, a few-cycle intense IR laser, together with four undulator sections and two dispersion sections. The wavelength of the UV seed laser is assumed to be $200 \mathrm{~nm}$ and that of the few-cycle IR laser is $800 \mathrm{~nm}$. We further assume that the lasers originate from the same Ti:sapphire oscillator which will allow tight synchronization between them. The schematic of the proposed scheme is shown in Fig. 1.

The first part of the proposed scheme is similar to the EEHG FEL $[18,19]$ in which the beam is energy modulated in the first modulator (M1) and then sent through a disper- 


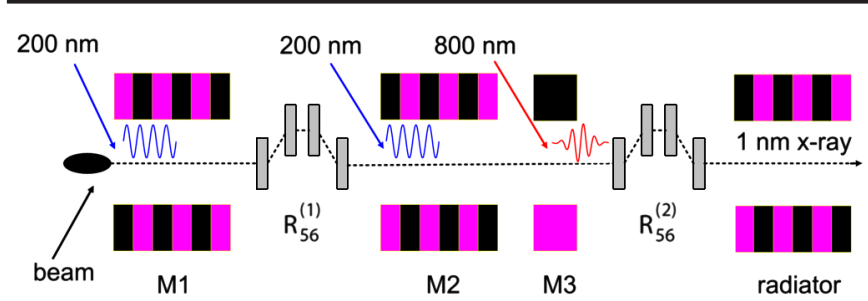

FIG. 1. (Color) Schematic of the proposed scheme for generation of isolated attosecond $\mathrm{x}$-ray pulse.

sion section with strong dispersion strength $R_{56}^{(1)}$ after which the modulation obtained in M1 is macroscopically washed out while simultaneously complicated fine structures (separated energy bands) are introduced into the phase space of the beam. In the EEHG scheme, a second laser is used to further modulate the beam energy in the second modulator (M2). After passing through the second dispersion section with dispersion strength $R_{56}^{(2)}$, the separated energy bands will convert to separated current bands and the echo signal then occurs as a recoherence effect caused by the mixing of the correlations between the modulation in the second modulator and the fine structures.

In our proposed scheme for generation of an attosecond x-ray pulse, we introduce an extra modulator (M3) between M2 and the second dispersion section. The beam interacts in M3 with a few-cycle intense laser of which the wavelength is chosen to be much longer than that of the laser in M2, so that part of the electrons around the zero crossing of the few-cycle laser gets almost linear energy chirp. With this additional energy chirp, the beam is longitudinally compressed after passing through the second dispersion section and the harmonic number is increased by the compression factor. As we will show below, in addition to assisting in extension of the harmonic number to a few hundred, the few-cycle laser also offers a possibility to select an isolated attosecond pulse.

We assume an initial Gaussian beam energy distribution with an average energy $E_{0}$ and the rms energy spread $\sigma_{E}$, and use the variable $p=\left(E-E_{0}\right) / \sigma_{E}$ for the dimensionless energy deviation of a particle. The initial normalized distribution function of the beam is $f_{0}(p)=$ $N(2 \pi)^{-1 / 2} e^{-p^{2} / 2}$, where $N$ is the number of electrons per unit length of the beam. The beam energy is modulated with the amplitude $\Delta E_{1}$ in $\mathrm{M} 1$, and the distribution function after beam passing through the first dispersion section with the dispersion strength $R_{56}^{(1)}$ is $[18,19]$

$$
f_{1}(\zeta, p)=\frac{N}{\sqrt{2 \pi}} \exp \left[-\frac{1}{2}\left[p-A_{1} \sin \left(\zeta-B_{1} p\right)\right]^{2}\right]
$$

where $A_{1}=\Delta E_{1} / \sigma_{E}, \zeta=k z, z$ is the longitudinal coordinate in the beam, $k$ is the wave number of the laser, and $B_{1}=R_{56}^{(1)} k \sigma_{E} / E_{0}$. The beam is then energy modulated in M2 with the dimensionless modulation amplitude $A_{2}$. Assuming the laser wavelength to be the same (in our example the laser wavelength is $200 \mathrm{~nm}$ ), at the exit of M2 the beam longitudinal phase space evolves to

$$
\begin{aligned}
f_{2}(\zeta, p)= & \frac{N}{\sqrt{2 \pi}} \exp \left[-\frac{1}{2}\left\{p-A_{2} \sin \zeta\right.\right. \\
& \left.\left.-A_{1} \sin \left[\zeta-B_{1}\left(p-A_{2} \sin \zeta\right)\right]\right\}^{2}\right]
\end{aligned}
$$

Before entering the second dispersion section, the beam is further energy modulated in M3 with an intense, fewcycle laser for which the wavelength is much longer than that in the upstream modulators. The carrier-envelope phase of the few-cycle laser pulse is set to $\pi / 2$ so that the oscillating electric field is zero at the pulse peak [20]. A snapshot of the $800 \mathrm{~nm}$ few-cycle laser field normalized to the peak value is shown in Fig. 2(a).

To show how the few-cycle laser assists in generation of much higher harmonic and an attosecond x-ray pulse, we add a linear energy chirp to the beam distribution before sending the beam to the second dispersion section. Assuming the linear energy chirp factor is $h=d p / d \zeta$, the resulting longitudinal phase space distribution at the exit from the second dispersion section is

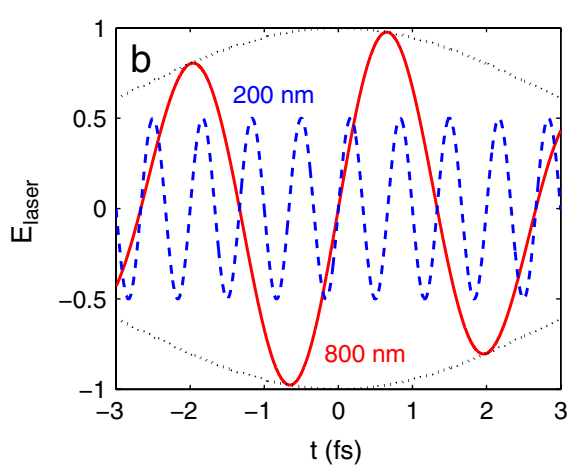

FIG. 2. (Color) (a) A snapshot of the laser field normalized to the peak value for the $800 \mathrm{~nm}$ few-cycle laser with pulse length 5 fs (FWHM of intensity). (b) Schematic of the synchronization between the laser in M2 and the few-cycle laser (laser field not in scale). 


$$
\begin{aligned}
f_{3}(\zeta, p)= & \frac{N}{\sqrt{2 \pi}} \exp \left[-\frac{1}{2}\left\{\left(1+h B_{2}\right) p-A_{2} \sin \left(\zeta-B_{2} p\right)\right.\right. \\
& -h \zeta-A_{1} \sin \left[\left(1+h B_{1}\right) \zeta\right. \\
& +A_{2} B_{1} \sin \left(\zeta-B_{2} p\right) \\
& \left.\left.\left.-\left(B_{1}+B_{2}+h B_{1} B_{2}\right) p\right]\right\}^{2}\right],
\end{aligned}
$$

where $B_{2}=R_{56}^{(2)} k \sigma_{E} / E_{0}$. Integration of Eq. (3) over $p$ yields the final current distribution which can be expanded in Fourier series,

$$
b(\zeta)=C N\left[1+2 \sum_{n=1}^{\infty} b_{n} \cos \left(C n \zeta+\psi_{n}\right)\right] .
$$

The wave number of the echo signal is $C n k$, where $C=$ $1 /\left(1+h B_{2}\right)$ is the compression factor and the corresponding maximized bunching factor for this harmonic radiation is

$$
\begin{aligned}
b_{n}= & \left|J_{n+1}\left(C n A_{2} B_{2}\right) J_{1}\left[A_{1}\left(C n B_{2}-B_{1}\right)\right]\right| \\
& \times \exp \left[-\frac{1}{2}\left(C n B_{2}-B_{1}\right)^{2}\right],
\end{aligned}
$$

The bunching factor at the harmonic number $m$ is defined as $\left\langle e^{i m k z}\right\rangle$, where $m k$ is the wave number of the harmonic radiation and the brackets denote averaging over the longitudinal coordinate $z$. A comparison between this equation and Eq. 6 in Ref. [19] indicates that for given energy modulation amplitudes the bunch compression ex- tends the harmonic number by a factor of $C$ while keeping the value of the bunching factor unchanged.

To show the basic process involved in our proposed scheme, let us consider the following case where the beam is compressed by a factor of 2 to generate the 40th harmonic. The dimensionless energy modulation amplitudes are assumed to be $A_{1}=3$ and $A_{2}=1$. Using the method described in Ref. [19], the required dispersion strengths and the chirp factor which maximize the bunching factor of the 40th harmonic $(n=20, C=2)$ are $B_{1} \approx$ $22.703, B_{2} \approx 0.581$, and $h \approx-0.861$. The phase space evolution is simulated with our $1 \mathrm{D}$ code which, though very simple, includes the key dynamics of the modulation and bunching processes.

The initial longitudinal phase space is shown in Fig. 3(a) where we assumed a longitudinally uniform beam with a Gaussian energy distribution. This is justified as long as the bunch length is much longer than the wavelength of the modulation. After interaction with the laser in M1, the beam is energy modulated and the longitudinal phase space evolves to Fig. 3(b). In HGHG scheme, the dispersion strength would be chosen $B_{1} \approx 1 / A_{1}$ to convert the energy modulation to density modulation after the beam passes through the dispersion section. For the EEHG scheme, as we stated earlier, the first dispersion section has a very strong dispersion strength (in our example it is about 2 orders of magnitude stronger than that in the HGHG scheme) so that after passing through it the energy modulation imprinted in M1 is washed out. This can be seen in
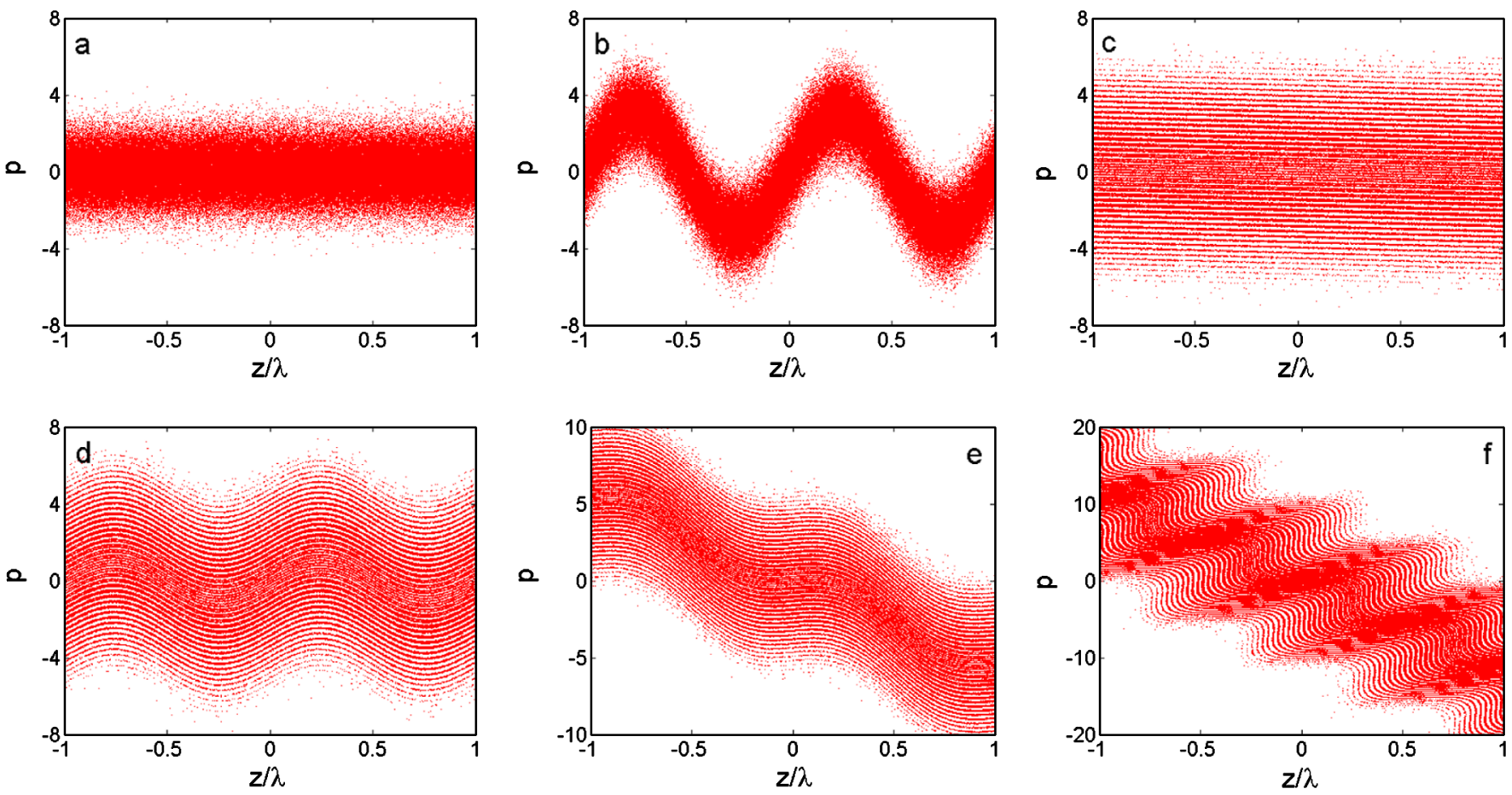

FIG. 3. (Color) Longitudinal phase space evolution: (a) the initial phase space; (b) after the first modulator; (c) after the first dispersion section; (d) after the second modulator; (e) after energy chirp is added; (f) at the exit from the second dispersion section. 
Fig. 3(c) where separated energy bands are generated in the phase space while no density modulation is present. The laser then interacts with the beam again in M2. Before sending the beam to the second dispersion section, we add a linear energy chirp to the beam, as is shown in Fig. 3(e). Finally, after passing through the second dispersion section, the separated energy bands convert to separated current bands and the beam is longitudinally compressed by a factor of 2 . The bunching factor for the 40th harmonic is found to be about 0.12 using the beam distribution in Fig. 3(f).

\section{GENERATION OF AN ISOLATED ATTOSECOND X-RAY PULSE}

To show the feasibility of the proposed scheme in generating an attosecond $\mathrm{x}$-ray pulse, we consider a representative set of parameters as listed in Table I. Our goal is to generate $1 \mathrm{~nm}$ radiation from a $200 \mathrm{~nm}$ UV seed laser.

A $200 \mathrm{~nm}$ laser with peak power $\sim 100 \mathrm{MW}$ is used to modulate the beam in M1 and M2 with the peak-to-peak energy modulation amplitude of $900 \mathrm{keV}$ which corresponds to $A_{1}=A_{2}=3$. To extend the harmonic number to 200 , we will use a larger compression factor as compared to the case in the last section. Assuming a compression factor of 10 , we find that the dispersion strengths and the chirp factor which maximize the bunching factor of the 200th harmonic $(n=20, C=10)$ are $B_{1} \approx 7.213, B_{2} \approx$ 0.039 , and $h \approx-23.241$, respectively. With these parameters, the electrons are tracked through the modulators and dispersion sections with our 1D code and the bunching factors for various harmonic numbers are calculated from definition and shown in Fig. 4 where it can be seen that the bunching factor for the 200th harmonic (1 nm wavelength for a $200 \mathrm{~nm}$ seeding laser) is about 0.12 . Sending this prebunched beam to the radiator will generate a powerful coherent x-ray at $1 \mathrm{~nm}$ wavelength.

To generate an isolated attosecond $\mathrm{x}$-ray pulse, an $800 \mathrm{~nm}$ few-cycle laser with energy $\sim 1 \mathrm{~mJ}$ and pulse length of $5 \mathrm{fs}$ (FWHM) is used to modulate the beam in M3. This kind of laser has been demonstrated with current technologies $[4,20]$. To take advantage of the bunch compression assisted harmonic generation, the few-cycle laser is time delayed to make its zero crossing overlap with that of the UV laser in M2, as is shown in Fig. 2(b). Because the

TABLE I. Main beam and undulator parameters.

\begin{tabular}{lc}
\hline \hline Electron beam energy & $3 \mathrm{GeV}$ \\
Peak current & $1 \mathrm{kA}$ \\
Normalized emittance & $1 \mathrm{~mm} \mathrm{mrad}$ \\
Slice energy spread & $150 \mathrm{keV}$ \\
Undulator period length in M1 and M2 & $25 \mathrm{~cm}$ \\
Number of undulator periods in M1 and M2 & 6 \\
Undulator period length in M3 & $20 \mathrm{~cm}$ \\
Number of undulator periods in M3 & 2 \\
\hline \hline
\end{tabular}

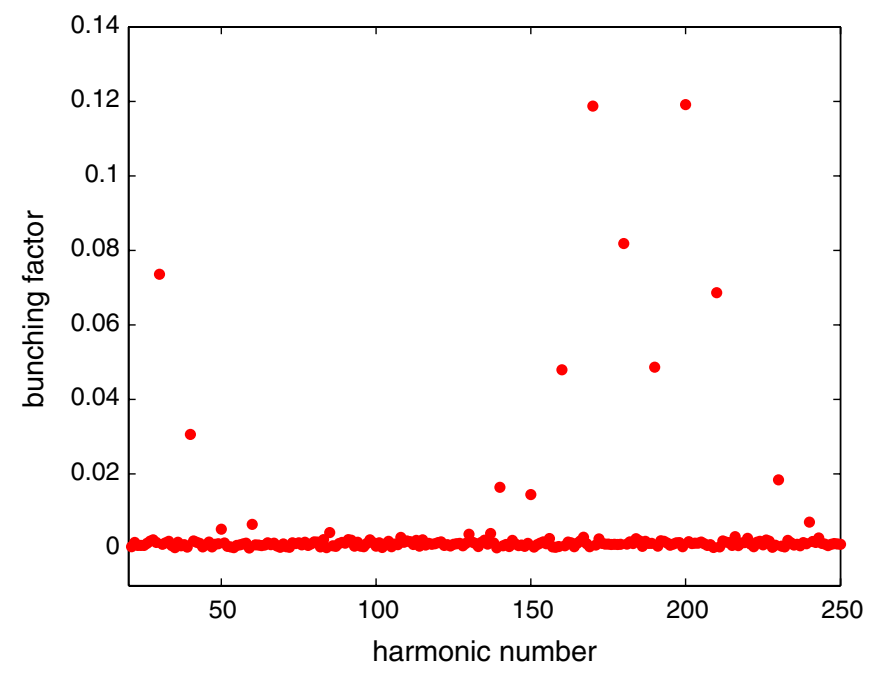

FIG. 4. (Color) Bunching factors for various compressed harmonic numbers $\mathrm{Cn}$.

wavelength of the few-cycle laser is much longer than that of the UV laser in M2, the electrons in the regions around the zero crossings get almost linear energy chirp which allows longitudinal compression after passing through a dispersive element. The longitudinal phase space of the beam after interaction with the few-cycle laser is simulated with our 1D code and shown in Fig. 5.

From Fig. 5 it follows that there are three regions where electrons have almost linear negative energy chirp: the region around the zero crossing of the central cycle $(t=$ 0 , region 1 in Fig. 5) and those around the zero crossings of the nearest side cycles $(t= \pm 2.67 \mathrm{fs}$, regions 2 and 3 in Fig. 5). The fine structures of the longitudinal phase space for the electrons at region 1 and region 2 are shown in Figs. 6(a) and 6(b) (the electrons in region 3 are similar to those in region 2). It can be easily seen that the energy

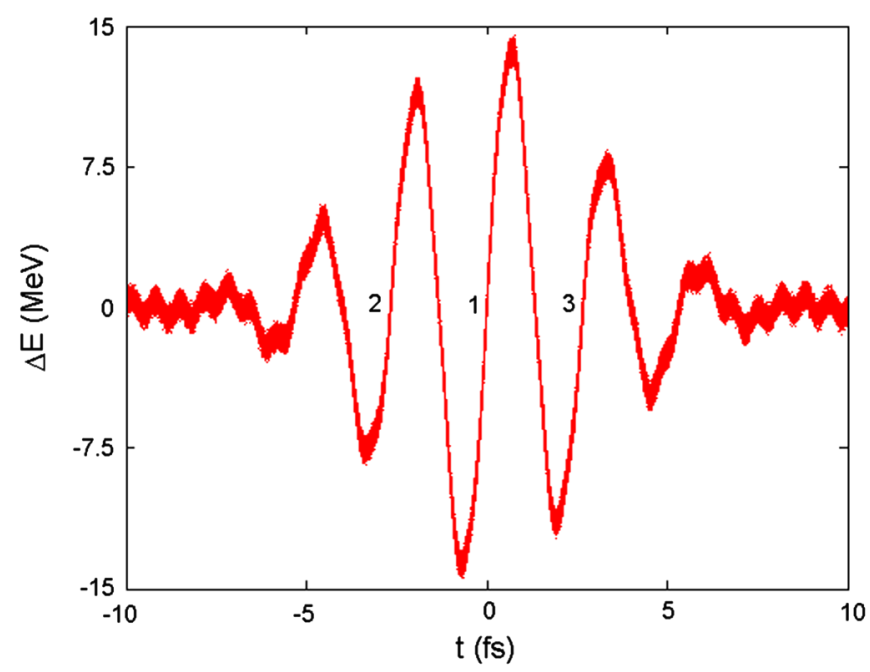

FIG. 5. (Color) Longitudinal phase space of the beam after interaction with the few-cycle laser (bunch head at left). 

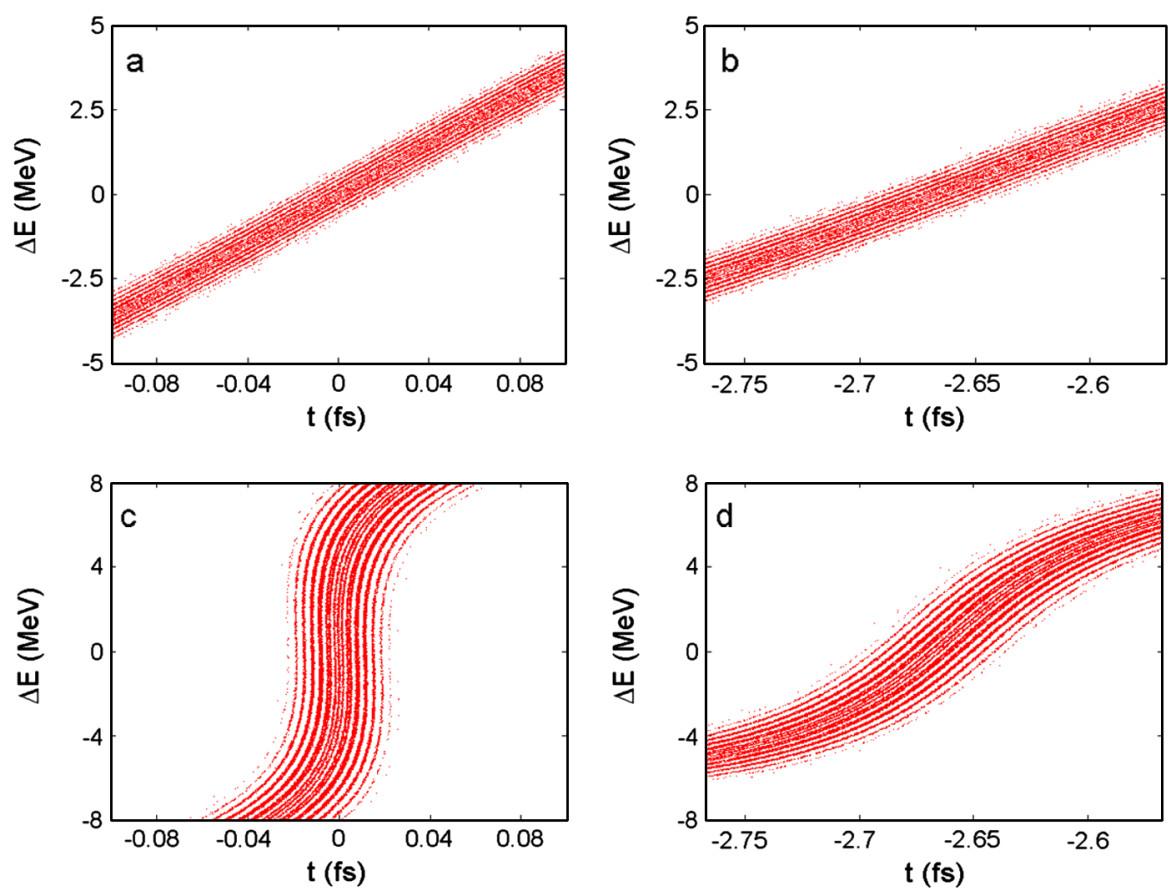

FIG. 6. (Color) Longitudinal phase space at the exit from M3 for the electrons in region 1-(a) and those in region 2-(b). Longitudinal phase space at the entrance to the radiator for the electrons in region $1-$ (c) and those in region 2 - (d).

chirp factor for the electrons in region 2 are approximately 1.5 times smaller than those in region 1 . The laser power is adjusted in such a way that only electrons in region 1 have the correct chirp factor $(h \approx-23.241)$ to maximize the 200th harmonic of the seed laser. With these conditions the longitudinal phase space of the electrons in these regions after passing through the second dispersion section are shown in Figs. 6(c) and 6(d).

As can be seen in Fig. 6, the separated energy bands in the central cycle are effectively converted to separated current bands which contain considerable higher harmonic components while those in the side cycles fail to convert to "upright" bands due to the small chirp factor. The bunching factor at $1 \mathrm{~nm}$ wavelength for the central slice is about 0.12 and that for all other slices is at the shot noise level, which makes possible the generation of an isolated attosecond $x$-ray pulse. The current of the central slice is also enhanced by a factor of 10 from the bunch compression. It can be expected that intense $1 \mathrm{~nm}$ x-rays will be generated after sending this prebunched, high-peak-current beam slice through the radiator.

Before proceeding to calculate the power and duration of the output $\mathrm{x}$-ray pulse, it is worth discussing the preservation of the "fine structures" in the phase space. These fine structures can be traced back to the separated energy bands generated after beam passes through the strong dispersion section. During passage through the dispersion sections and the modulators, the coherent and incoherent synchrotron radiation (CSR and ISR) will have some smearing effects on the fine structures. Quantum fluctuations in the process of ISR lead to diffusion in energy. If the rms value of the energy spread caused by this diffusion exceeds the spacing of two adjacent energy bands, it may result in the overlapping of the bands, which will smear the fine structures of the longitudinal phase space. The $z$-dependent CSR wake also introduces an additional energy modulation inside the dispersive section, which may result in distortion of the echo modulation.

Analysis shows that the spacing of the two adjacent energy bands is about $\left(\pi / B_{1}\right) \sigma_{E}$ which in our example is about $65 \mathrm{keV}$. The energy spread introduced by passing a length $L$ in a bend with the bending radius $\rho$ is [21]

$$
\left.\Delta \sigma_{E}^{2}\right|_{\mathrm{ISR}}=\frac{55 e^{2} \hbar c}{48 \sqrt{3}} \frac{L}{\rho^{3}} \gamma^{7}
$$

where $\gamma$ is the relativistic factor. Equation (6) implies that the diffusion caused by ISR strongly depends on the design of the dispersion section and we expect it to be more severe for the first dispersion section which has the largest value of $R_{56}$. Here we consider a representative dispersion section consisting of a symmetric four-dipole chicane with a dispersion strength of $4.6 \mathrm{~mm}$ (corresponding to $B_{1}=$ 7.213). The length of each dipole is $50 \mathrm{~cm}$ and the distances between the first and the second dipoles and that between the third and the fourth dipoles are both $60 \mathrm{~cm}$. The bending angle is about 2.8 degrees. We found that the ISR induced energy spread is about $9.4 \mathrm{keV}$, which is much smaller than the spacing of the separated energy bands. So it should not have significant effects on the fine structures.

The CSR effect in the first dispersion section should be negligible, because there is no density modulation in the 
chicane. As for the second dispersion section, due to the presence of $R_{51}$, the bunching is also smeared out everywhere inside the chicane and it only occurs at the very end of the last dipole where $R_{51} \sigma_{x}$ is much smaller than the wavelength of the generated harmonic. Considering the fact that the energy change in the 4th dipole only slightly affects the longitudinal position of the particle, the CSR effect does not seem significant to affect the fine structures.

It is worth pointing out that the separated energy bands should also be preserved during the energy modulation in M2 and M3. The undulator radiation also causes slice energy spread growth. For a planar undulator, the slice energy spread growth from quantum diffusion can be calculated as [22]

$$
(\Delta \gamma)^{2}=\frac{7}{15} \frac{\hbar}{m_{0} c} L_{u} r_{e} \gamma^{4} \kappa_{\omega}^{3} K^{2} F(K),
$$

where $r_{e}$ is the classical radius of the electron, $\kappa_{\omega}=$ $2 \pi / \lambda_{\omega}, \lambda_{\omega}$ is the undulator period length, $L_{u}$ is the total length of the undulator, $K$ is the undulator strength, and $F(K) \approx 1.42 K$ when $K \gg 1$. The energy spread growth for our example is found to be about $2.5 \mathrm{keV}$ in M2 and $6.0 \mathrm{keV}$ in $\mathrm{M} 3$ which are also much smaller than the spacing of the adjacent energy bands.

Another issue of concern is the second order temporal dispersion from the electron beam divergence. The separation of the fine structures in the longitudinal direction is on the order of the UV laser wavelength before M2, but it reduces to about $1 \mathrm{~nm}$ after $\mathrm{M} 2$. So it is most vulnerable in the second dispersion section. The second order transport matrix elements $T_{522}$ and $T_{544}$ are about $L_{c} / 2$, where $L_{c}$ is the total length of the second chicane. Note that the dispersion strength is very small for the second dispersion section so that $L_{c}$ can be smaller than $1 \mathrm{~m}$, and the beam divergence is typically on the order of a few microradians; we conclude that the smearing effect from the beam divergence should not significantly affect the fine structures.

Once these fine structures are preserved during the transport, one can expect intense $\mathrm{x}$-ray radiation after sending the beam to the radiator. The output radiation field may be calculated by summing up the field of each electron in the radiator. The output $\mathrm{x}$-ray pulse length is determined by both the electron bunch length and the slippage length in the undulator. For the SASE schemes [7,9-14], the slippage length associated with the long FEL undulator typically limits the radiation pulse length to a few hundred attoseconds. Since in our scheme a short section of the beam is effectively prebunched at the radiator entrance, only a short undulator is required to generate intense coherent $\mathrm{x}$-ray radiation which may allow pushing the pulse duration to below the atomic unit of time ( $\sim 24$ as). In our example, we used a planar undulator with $N_{u}=12$ periods and the period length $\lambda_{u}=4 \mathrm{~cm}$ to generate $\lambda_{r}=1 \mathrm{~nm}$ x rays. We further assumed that the relative longitudinal position of the electrons did not

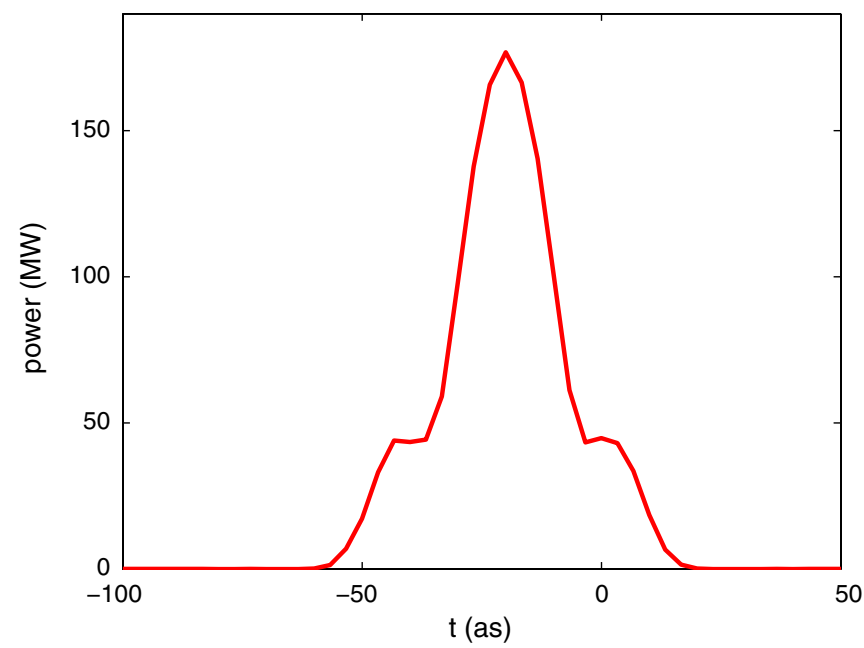

FIG. 7. (Color) Power of the generated $1 \mathrm{~nm} x$-ray pulse. The pulse length is 20 as (FWHM).

change during the passage through the short radiator (i.e. no FEL interaction), which was justified for an electron beam with $1 \mu \mathrm{m}$ normalized emittance and a transverse rms size $\sigma_{x}=20 \mu \mathrm{m}$. For these parameters, the singleelectron undulator radiation has a rms transverse size $\sqrt{2 \lambda_{r} \lambda_{u} N_{u}} /(4 \pi) \ll \sigma_{x}$ and can be approximated as a plane wave. Thus, the output power can be calculated as (see for example [23])

$$
P(t)=\frac{e^{2} c^{2} Z_{0} K^{2}[J J]^{2} \lambda_{u}^{2}}{32 \pi \sigma_{x}^{2} \gamma_{0}^{2} \lambda_{r}^{2}}\left|\sum_{j} e^{-i 2 \pi c\left(t_{j}-t\right) / \lambda_{r}}\right|^{2},
$$

where $Z_{0}=377 \Omega$ is the vacuum impedance, $[J J]=$ $J_{0}(\xi)-J_{1}(\xi)$ is the usual Bessel function factor associated with the planar undulator, $\xi=K^{2} /\left(4+2 K^{2}\right), \quad E_{0}=$ $\gamma_{0} m c^{2}$ is the beam energy, and the sum over the $j$ th electron is carried out within the radiation slippage length, i.e. $0<c\left(t_{j}-t\right)<N_{u} \lambda_{r}$.

Using the beam distribution at the radiator entrance, the power profile of the $\mathrm{x}$-ray radiation at $1 \mathrm{~nm}$ wavelength generated in the radiator is shown in Fig. 7. The peak power is about $180 \mathrm{MW}$, and the pulse length is about 20 as (FWHM), shorter than the atomic unit of time. It should be pointed out that the bunching factor depends on the energy chirp factor which varies if the IR laser energy changes. In order to keep the fluctuation of the output Xray power smaller than $10 \%$, the laser energy fluctuation should be controlled to be within $0.5 \%$.

\section{CONCLUSIONS}

In conclusion, we have proposed a scheme that combines the EEHG technique with bunch compression which extends the harmonic numbers to a few hundred. The required energy chirp is generated using a few-cycle intense laser which also assists in selection of an isolated attosecond $\mathrm{x}$-ray pulse. We emphasize that this pulse is 
only generated due to created microstructures in the beam, and does not involve additional bunching due to the FEL interaction. Using a representative realistic set of parameters, we have shown that $1 \mathrm{~nm}$ x-ray pulse with peak power of a few hundred MW and duration shorter than the atomic unit of time can be generated from a $200 \mathrm{~nm}$ ultraviolet seed laser. It is capable of breaking the 24 as time barrier and may open a new regime of ultrafast sciences, such as realtime observation and control of atomic-scale electron dynamics, study of short-lived transit states for atoms and molecules, etc.

\section{ACKNOWLEDGMENTS}

We thank A. Chao, Y. Ding, D. Ratner, and J. Wu for helpful discussions. This work was supported by the U.S. Department of Energy under Contract No. DE-AC0276 SF00515.

[1] P. M. Paul, E. S. Toma, P. Breger, G. Mullot, F. Augé, Ph. Balcou, H. G. Muller, and P. Agostini, Science 292, 1689 (2001).

[2] E. Goulielmakis, M. Uiberacker, R. Kienberger, A. Baltuska, V. Yakovlev, A. Scrinzi, Th. Westerwalbesloh, U. Kleineberg, U. Heinzmann, M. Drescher, and F. Krausz, Science 305, 1267 (2004).

[3] M. Uiberacker, Th. Uphues, M. Schultze, A. J. Verhoef, V. Yakovlev, M.F. Kling, J. Rauschenberger, N.M. Kabachnik, H. Schröder, M. Lezius, K. L. Kompa, H.-G. Muller, M. J. J. Vrakking, S. Hendel, U. Kleineberg, U. Heinzmann, M. Drescher, and F. Krausz, Nature (London) 446, 627 (2007).

[4] F. Krausz and M. Ivanov, Rev. Mod. Phys. 81, 163 (2009).

[5] E. Goulielmakis, M. Schultze, M. Hofstetter, V.S. Yakovlev, J. Gagnon, M. Uiberacker, A. L. Aquila, E. M. Gullikson, D. T. Attwood, R. Kienberger, F. Krausz, and U. Kleineberg, Science 320, 1614 (2008).

[6] E. L. Saldin, E. A. Schneidmiller, and M. V. Yurkov, Opt. Commun. 212, 377 (2002).
[7] P. Emma, K. Bane, M. Cornacchia, Z. Huang, H. Schlarb, G. Stupakov, and D. Walz, Phys. Rev. Lett. 92, 074801 (2004).

[8] A. A. Zholents and W. M. Fawley, Phys. Rev. Lett. 92, 224801 (2004).

[9] E. L. Saldin, E. A. Schneidmiller, and M. V. Yurkov, Opt. Commun. 239, 161 (2004).

[10] A. A. Zholents and G. Penn, Phys. Rev. ST Accel. Beams 8, 050704 (2005).

[11] E. L. Saldin, E. A. Schneidmiller, and M. V. Yurkov, Phys. Rev. ST Accel. Beams 9, 050702 (2006).

[12] Y. Ding, Z. Huang, D. Ratner, P. Bucksbaum, and H. Merdji, Report No. SLAC-PUB-13393 (2008).

[13] A. A. Zholents and M.S. Zolotorev, New J. Phys. 10, 025005 (2008).

[14] S. Reiche, P. Musumeci, C. Pellegrini, and J. B. Rosenzweig, Nucl. Instrum. Methods Phys. Res., Sect. A 593, 45 (2008).

[15] N. R. Thompson and B. W. J. McNeil, Phys. Rev. Lett. 100, 203901 (2008).

[16] L.-H. Yu, M. Babzien, I. Ben-Zvi, L. F. DiMauro, A. Doyuran, W. Graves, E. Johnson, S. Krinsky, R. Malone, I. Pogorelsky, J. Skaritka, G. Rakowsky, L. Solomon, X. J. Wang, M. Woodle, V. Yakimenko, S. G. Biedron, J. N. Galayda, E. Gluskin, J. Jagger, V. Sajaev, and I. Vasserman, Science 289, 932 (2000).

[17] X. J. Wang, Y. Shen, T. Watanabe, J. B. Murphy, J. Rose, and T. Tsang, in Proceedings of FEL 2006 (BESSY, Berlin, 2006), p. 18.

[18] G. Stupakov, Phys. Rev. Lett. 102, 074801 (2009).

[19] D. Xiang and G. Stupakov, Phys. Rev. ST Accel. Beams 12, 030702 (2009).

[20] A. Baltuška, Th. Udem, M. Uiberacker, M. Hentschel, E. Goulielmakis, Ch. Gohle, R. Holzwarth, V.S. Yakovlev, A. Scrinzi, T. W. Hänsch, and F. Krausz, Nature (London) 421, 611 (2003).

[21] A. W. Chao and M. Tigner, Handbook of Accelerator Physics and Engineering (World Scientific, Singapore, 2006), 3rd ed.

[22] E. Saldin, E. A. Schneidmiller, and M. V. Yurkov, Nucl. Instrum. Methods Phys. Res., Sect. A 381, 545 (1996).

[23] Z. Huang and K. Kim, in Proceedings of the 18th Particle Accelerator Conference, New York, 1999 (IEEE, New York, 1999), p. 2495. 